\title{
The Role of NADPH Oxidase in Chronic Intermittent Hypoxia-Induced Pulmonary Hypertension in Mice
}

\author{
Rachel E. Nisbet ${ }^{1 *}$, Anitra S. Graves ${ }^{1 *}$, Dean J. Kleinhenz' ${ }^{1}$ Heidi L. Rupnow1, Alana L. Reed', \\ Tai-Hwang M. Fan' ${ }^{1}$, Patrick O. Mitchell ${ }^{1}$, Roy L. Sutliff ${ }^{1}$, and C. Michael Hart ${ }^{1}$ \\ ${ }^{1}$ Department of Medicine, Atlanta Veterans Affairs and Emory University Medical Centers, Atlanta, Georgia
}

\begin{abstract}
Obstructive sleep apnea, characterized by intermittent periods of hypoxemia, is an independent risk factor for the development of pulmonary hypertension. However, the exact mechanisms of this disorder remain to be defined. Enhanced NADPH oxidase expression and superoxide $\left(\mathrm{O}_{2}^{-}\right.$.) generation in the pulmonary vasculature play a critical role in hypoxia-induced pulmonary hypertension. Therefore, the current study explores the hypothesis that chronic intermittent hypoxia (CIH) causes pulmonary hypertension, in part, by increasing NADPH oxidase-derived reactive oxygen species (ROS) that contribute to pulmonary vascular remodeling and hypertension. To test this hypothesis, male $\mathrm{C} 57 \mathrm{BI} / 6$ mice and gp91phox knockout mice were exposed to $\mathrm{CIH}$ for 8 hours per day, 5 days per week for 8 weeks. CIH mice were placed in a chamber where the oxygen concentration was cycled between $21 \%$ and $10 \% \mathrm{O}_{2}$ 45 times per hour. Exposure to $\mathrm{CIH}$ for 8 weeks increased right ventricular systolic pressure (RVSP), right ventricle (RV):left ventricle (LV) + septum (S) weight ratio, an index of RV hypertrophy, and thickness of the right ventricular anterior wall as measured by echocardiography. CIH exposure also caused pulmonary vascular remodeling as demonstrated by increased muscularization of the distal pulmonary vasculature. $\mathrm{ClH}$-induced pulmonary hypertension was associated with increased lung levels of the NADPH oxidase subunits, Nox4 and p22phox, as well as increased activity of platelet-derived growth factor receptor $\beta$ and its associated downstream effector, Akt kinase. These $\mathrm{CIH}$-induced derangements were attenuated in similarly treated gp91phox knockout mice. These findings demonstrate that NADPH oxidase-derived ROS contribute to the development of pulmonary vascular remodeling and hypertension caused by $\mathrm{CIH}$.
\end{abstract}

Keywords: hypoxia; pulmonary hypertension; nitric oxide; NADPH oxidase

Sleep-disordered breathing encompasses a spectrum of conditions characterized, in part, by repetitive perturbations in airflow and oxygenation. Obstructive sleep apnea (OSA), the most prevalent of these disorders, affects $2 \%$ of women and $4 \%$ of men in the United States (1). Patients with OSA have higher rates of systemic hypertension (2-7), coronary atherosclerotic heart disease $(8,9)$, heart failure (10-12), cardiac arrhythmias $(5)$, and stroke (2). However, the association between sleep apnea and pulmonary hypertension remains less certain. Clinical evidence in patients with OSA without underlying lung disease

(Received in original form April 11, 2008 and in final form September 10, 2008)

* These two authors contributed equally to this work.

This work was supported in part by grants HL-070892 (R.L.S.) and DK-074518 (C.M.H.) from the National Institutes of Health and by a Merit Review Award (C.M.H) from the Veterans Affairs Research Service.

Correspondence and requests for reprints should be addressed to $\mathrm{C}$. Michael Hart, M.D., Professor of Medicine, Division of Pulmonary and Critical Care Medicine, Atlanta VAMC (151-P), 1670 Clairmont Rd., Decatur, GA 30033. E-mail: Michael.hart3@va.gov

Am J Respir Cell Mol Biol Vol 40. pp 601-609, 2009

Originally Published in Press as DOI: 10.1165/rcmb.2008-0145OC on October 23, 2008

Internet address: www.atsjournals.org

\section{CLINICAL RELEVANCE}

Chronic intermittent hypoxia $(\mathrm{CIH})$-induced alterations in NADPH oxidase-derived reactive oxygen species (ROS) contribute to the development of pulmonary hypertension and vascular remodeling. NADPH oxidase-derived ROS represent potential novel targets for intervention in OSAassociated vascular derangements.

indicates a prevalence of diurnal pulmonary hypertension of 20 to $41 \%(8,10,13,14)$. Pulmonary artery pressure increases acutely in response to apneas and/or hypopneas in patients with OSA (15). Furthermore, patients with severe OSA can develop sustained pulmonary hypertension, even in the absence of other cardiac or lung diseases (9). Treatment with continuous positive airway pressure (CPAP) reduces pulmonary artery pressures in patients with OSA $(14,16)$ and attenuates pulmonary vascular resistance caused by hypoxemia (16). Collectively, these observations suggest that intermittent hypoxia increases pulmonary artery pressure and contributes to vascular remodeling, and ultimately, persistent pulmonary hypertension.

The mechanisms that link OSA with vascular dysfunction are unknown. OSA is associated with several physiological derangements that might contribute to vascular dysfunction, including hypoxemia, hypercapnia, arousals, upper airway obstruction, and increased sympathetic tone (5). Pulmonary hypertension, characterized by pulmonary vasoconstriction, vascular remodeling, and endothelial dysfunction, constitutes a prominent vascular derangement in mouse models of chronic intermittent hypoxia (CIH) $(17$, 18). Therefore, in the current study, we employed a mouse model of $\mathrm{CIH}$ to determine mechanisms of pulmonary hypertension caused by repetitive reductions in nocturnal oxygen saturation.

Recent evidence suggests that hypoxia-induced activation of NADPH oxidase plays an important role in pulmonary hypertension in the mouse $(19,20)$. For example, in isolated-perfused lung preparations from wild-type mice, exposure to $3 \%$ oxygen caused acute vasoconstrictor responses that were blunted in mice lacking the NADPH oxidase subunit p47phox (21). Similarly, C57B1/6 mice exposed to chronic hypoxia $(10 \%$ oxygen) for 3 weeks demonstrated increased superoxide generation in pulmonary arteries and increased right ventricular pressure and pulmonary arterial medial wall thickness (22). These chronic hypoxia-induced derangements were completely abolished in similarly treated mice lacking the gp91phox NADPH oxidase subunit (22). The role of NADPH oxidase in pulmonary hypertension was also supported by recent evidence that chronic hypoxia increased expression of the Nox4 NADPH oxidase subunit in mice (23). NADPH oxidase is an important source of superoxide production in the vasculature. Superoxide generation in the vascular wall is a wellrecognized stimulus for vascular wall cell proliferation and vasoconstriction (24). Superoxide also reacts rapidly with NO to reduce NO bioavailability (25), thus contributing to the 
pathogenesis of pulmonary hypertension by promoting an imbalanced production of vasodilatory and vasoconstrictive mediators leading to endothelial dysfunction. These reports indicate that NADPH oxidase is an important mediator of hypoxia-induced pulmonary hypertension and that it contributes to pulmonary vasoconstriction and vascular remodeling. Therefore, the current study was designed to determine if $\mathrm{CIH}$ altered NADPH oxidase expression, superoxide generation, and NO bioavailability to cause pulmonary hypertension.

\section{MATERIALS AND METHODS}

\section{Mouse Model of Chronic Intermittent Hypoxia}

Male C57B1/6 mice and gp91phox knockout mice (8-10 wk old) were purchased from the Jackson Laboratory (Bar Harbor, ME). The mice were divided into two groups: Control (room air) and CIH. All animals had access to standard mouse chow and water, ad libitum, during exposure to Control and $\mathrm{CIH}$ conditions. All animal studies were reviewed and approved by the Institutional Animal Care and Use Committee of the Atlanta VA Medical Center.

Control and CIH mice were weighed weekly and housed in standard shoebox cages with five mice per cage. During $\mathrm{CIH}$ exposure, the standard cage lid of selected animals was replaced with a customized Plexiglas lid for 8 hours per day (during the rodent nocturnal cycle), 5 days per week for 8 weeks. The lid of the CIH chamber was connected to a gas reservoir that was separated from the mouse chamber by a time-delayed, electronic switch-operated sliding door. While the door was in the "open" position, the reservoir filled with $100 \%$ nitrogen, and at the same time, a portal to room air permitted mixing of gas in the animal chamber with room air. Two small silent fans were incorporated into the customized lid to facilitate rapid mixing of gas in the animal chamber with nitrogen in the reservoir or with room air. When activated, the door moved into the "closed" position, thereby initiating equilibration of the gas in the animal chamber with that in the nitrogen reservoir while simultaneously obstructing the communication of gas in the animal chamber with room air. An oxygen sensor (Model \#S-450 OX; AIM Co., Lacuna, NY) was placed inside the $\mathrm{CIH}$ chamber and the door actuation adjusted to produce decreases in oxygen tension within the chamber to $10 \%$ every 90 seconds. The nadir in oxygen tension $(10 \%)$ within the chamber during each cycle was followed by restoration of oxygen tension to $21 \%$ by flushing the animal chamber with room air. Alterations in oxyhemoglobin saturation of mice in the $\mathrm{CIH}$ chamber were monitored with a pulse oximeter specifically designed for rodents (MouseOX; Starr Life Sciences, Oakinant, PA). In preliminary studies, to control for potential confounding effects caused by operation of the customized lid, selected animals were housed in the CIH chamber, exposed to the operation of the customized lid as described above, but exposed to room air rather than hypoxia by filling the reservoir with $21 \%$ oxygen rather than nitrogen. The pulmonary hemodynamic findings in these animals were indistinguishable from those control animals exposed to room air and housed in cages with standard lids (data not shown).

\section{Assessment of CIH Effects on Cardiac Chamber Size and Right Ventricular Systolic Pressure}

To determine if exposure to $\mathrm{CIH}$ caused right ventricular hypertrophy, mice were killed with $\mathrm{CO}_{2}$, and the hearts were removed and weighed. The free wall of the right ventricle (RV) was then carefully dissected from the left ventricle (LV) and septum (S), and each was individually weighed to permit calculation of the $\mathrm{RV}: \mathrm{LV}+\mathrm{S}$ weight ratio as an index of right ventricular hypertrophy. Right ventricular systolic pressure (RVSP) was measured in mice lightly anesthetized with isoflurane. A 0.8-F microtip pressure transducer (Millar Instruments, Houston, TX) was inserted into the surgically exposed right jugular vein and advanced into the right ventricle (RV). Right ventricular pressure was continuously monitored for 10 minutes, and data were analyzed using a Powerlab system (AD Instruments, Denver, CO). RVSP measurements were recorded in the morning after overnight exposure to room air to minimize the acute effects of hypoxia on the pulmonary vasculature.

\section{Transthoracic Echocardiography}

To further assess the temporal onset of pulmonary hypertension in our model during $\mathrm{CIH}$ exposure, transthoracic echocardiograms (TTEs) were performed on mice using a Vevo 770 High-Resolution In Vivo Imaging System (VisualSonics, Toronto, ON, Canada) equipped with a RMV 707B High-Frame-Rate Scanhead (frequency band 15$45 \mathrm{MHz}$ ) and a SONOS 5500 ultrasound unit (Philips Medical Systems, Bothell, WA) equipped with a $15-\mathrm{MHz}$ linear-array transducer and a $12-\mathrm{MHz}$ phase-array transducer. Echocardiograms were acquired before $\mathrm{CIH}$ exposure (baseline), then at 2-week intervals for 8 weeks. During echocardiography, the animals were lightly anesthetized with $1 \%$ isoflurane, and the body temperature was continuously monitored using a rectal thermometer probe to maintain body temperature at 36 to $37^{\circ} \mathrm{C}$. Under these conditions, the animal's heart rate could be maintained between 400 and 550 beats per minute. Two-dimensional and M-mode echocardiography were used to assess wall motion, chamber dimensions, and wall thickness and to calculate the fractional shortening. Color flow Doppler was used to assess valve functions. Images were obtained from the parasternal long axis, the parasternal short axis (mid-papillary muscle level), the apical four-chamber, the apical two-chamber, and the apical three-chamber views.

\section{Immunohistochemical and Morphometric Analysis}

Lungs were perfused blood-free, then perfused with and embedded in optimal cutting temperature (OCT) compound and frozen at $-80^{\circ} \mathrm{C}$. Sections $(5 \mu \mathrm{m})$ from the lungs were fixed in $4 \%$ formaldehyde, washed three times ( 5 min each) in PBS, and endogenous peroxidase activity was quenched with $3 \% \mathrm{H}_{2} \mathrm{O}_{2}$ in PBS. Sections were permeabilized with $0.05 \%$ Tween-20 (PBS-T), blocked with $5 \%$ donkey serum and incubated overnight at $4^{\circ} \mathrm{C}$ with rabbit anti- $\alpha$-smooth muscle actin $(\alpha-$ SMA) antibody (LabVision Coporation, Fremont, CA). Sections were then incubated with biotinylated donkey anti-rabbit secondary antibody (Jackson Immunoresearch Laboratories, West Grove, PA) followed by horseradish peroxidase-streptavidin (Vectastain kit, Vector Laboratories, Burlingame, CA). Color was developed with 3,3'diaminobenzidine tetrahydrochloride substrate (Vector Laboratories), and sections were counterstained with hematoxylin and coverslipped. Rabbit IgG was used to control for nonspecific antibody binding. Multiple high-power photomicrographs were obtained using a Leica DM4000B microscope (Bannockburn, IL). In each section, the number of $\alpha$-SMA-positive blood vessels associated with intra-acinar airways was counted per high-power field, and vessels with lumenal diameters less than $100 \mu \mathrm{m}$ were subjected to digital morphometric analyses. Lumenal circumference (LC) and outer vessel circumference (OC) were determined from $\alpha$-SMA-positive vessels using Scion Image Software (Scion Image Beta 4.0.2; Scion Corporation, Frederick, MD). The following formulae were used: lumen cross-sectional area = $(\mathrm{LC})^{2} / 4 \pi$, muscular wall thickness $=[(\mathrm{OC} / 2 \pi)-(\mathrm{LC} / 2 \pi)]$.

\section{Nitrosyl-Hemoglobin (NO-Hgb) Measurements}

At the time mice were killed, blood was aspirated via cardiac puncture using a heparinized 1-cc syringe. After centrifugation at 2,000 $\times \mathrm{g}$ for 10 minutes at $4^{\circ} \mathrm{C}$, serum was aspirated, an equal volume of PBS, preaerated with nitrogen for $\geqslant 20$ minutes, was mixed with the remaining red blood cells (RBCs) and snap-frozen in liquid nitrogen. Measurements of NO-Hgb were performed using an EMX electron spin resonance (ESR) spectrometer (Bruker, Karlruhe, Germany) with a super-high Q microwave cavity as recently reported $(27,28)$. The ESR settings for detection of NO-Hgb were: field sweep, $300 \mathrm{G}$; microwave frequency, $9.78 \mathrm{GHz}$; microwave power, $10 \mathrm{~mW}$; modulation amplitude, $3 \mathrm{G}$; conversion time, 2,624 ms; time constant, 5,248 ms; receiver gain, $1 \times 10^{5}(28)$.

\section{Western Blot Analysis}

Immediately after killing, homogenates of peripheral lung tissue were prepared and their protein concentrations determined as previously reported (29). Whole lung homogenates (45 $\mu \mathrm{g}$ protein/lane) were subjected to SDS-PAGE (4-12\% gradient gels) (Invitrogen, Carlsbad, CA) followed by electroblotting of proteins onto polyvinylidene fluoride (PVDF) or nitrocellulose (NC) membranes. After appropriate blocking (5\% nonfat dried milk or $3 \%$ bovine serum albumin [BSA]), the blots were probed with primary antibodies $(1: 1,000)$ specific to 
endothelial nitric oxide synthase (eNOS) (BD Transduction Laboratories, San Jose, CA), platelet-derived growth factor (PDGF) receptor $\beta$ (PDGFR $\beta$ ), phosphorylated PDGFR $\beta$ (P-PDGFR $\beta$ ), phosphatase and tensin homolog deleted on chromosome 10 (PTEN), actin (Santa Cruz Biotechnology, Santa Cruz, CA), Nox4 (gift from Dr. David Lambeth, Emory University), PPAR $\gamma$ (Bethyl Laboratories, Montgomery, TX), Akt, or phospho-Akt (Ser473 and Thr308) (Cell Signaling Technology, Danvers, MA) in 5\% powdered nonfat dry milk or 3\% BSA on a rocking platform overnight at $4^{\circ} \mathrm{C}$. After washing, membranes were incubated with horseradish peroxidase-conjugated secondary antibody (Jackson ImmunoResearch). Levels of proteins were normalized to the actin content of the same sample.

For the analysis of remaining NADPH oxidase subunits, lung homogenates $(500 \mu \mathrm{g}$ protein) were incubated with $5 \mu \mathrm{g}$ of antibodies directed against subunits of NADPH oxidase, including p22phox (gift from Dr. Mark Quinn, Montana State University), p47phox (BD Transduction Laboratories), gp91phox, and p67phox (Santa Cruz Biotechnology), on a rocking platform overnight at $4^{\circ} \mathrm{C}$ as we have recently reported (29). Antibody-NADPH oxidase subunit complexes were collected by incubation with GammaBind sepharose beads (Amersham Biosciences, Piscataway, NJ), and the immunocomplexes were precipitated by centrifugation. Immunoprecipitated proteins were then separated with SDS-PAGE, transferred to PVDF membranes, and immunoblotted for the appropriate NADPH oxidase subunit as previously described. For all blots, immunodetection was performed using a chemiluminescence method (SuperSignal; Pierce Biotechnology, Rockford, IL), and relative immunoreactive levels of proteins were quantified using the ChemiDoc XRS imaging system and Quantity One software (version 4.5; Bio-Rad Laboratories, Hercules, CA).

\section{Quantitative Real-Time PCR}

Real-time PCR was performed to quantify mRNA levels of Nox4, p22phox, p47phox, p67phox, and gp91phox. Isolation of total RNA from whole lung homogenates was performed according to the manufacturer's protocol (RNeasy Mini Kit; Qiagen, Valencia, CA). Total RNA was reverse transcribed using random primers and a SuperScript II kit (Invitrogen, San Diego, CA). The first-strand cDNA was purified using a microbiospin 30 column (Bio-Rad Laboratories) in Tris buffer and then stored at $-80^{\circ} \mathrm{C}$ until used. Lung cDNA was amplified using a LightCycler real-time thermocycler (Roche Diagnostics Corp, Indianapolis, IN). The mRNA copy numbers were calculated from standard curves generated from rat p22phox, p47phox, p67phox, mouse gp91phox, and 18S templates. None of the treatment conditions had a significant effect on $18 \mathrm{~S}$ expression.

\section{Superoxide $\left(\mathrm{O}_{2}^{-} \cdot\right)$ Production in Lung Tissue}

$\mathrm{O}_{2}^{-}$. generation in intact lung tissue was examined in frozen sections from Control or $\mathrm{CIH}$ lungs using dihydroethidium as we have previously reported (29). Lungs were perfused blood-free, then perfused with and embedded in OCT compound and frozen at $-80^{\circ} \mathrm{C}$. Sections $(35 \mu \mathrm{m})$ were then prepared and stained with dihydroethidium (DHE, $10 \mu \mathrm{M})$ by covering the section with $30 \mu \mathrm{l}$ of DHE and a coverslip followed by incubation at $37^{\circ} \mathrm{C}$ in a humidified, $5 \% \mathrm{CO}_{2}$ atmosphere for 30 minutes. Sections from each treatment group were examined by fluorescence microscopy, and images were acquired at $\times 40$ magnification using identical instrument settings. To quantify superoxide production in lung tissue, fresh pieces of lung tissue were incubated for 60 minutes at $37^{\circ} \mathrm{C}$ in 1-hydroxy-3-methoxycarbonyl-2,2,5,5-tetramethylpyrrolidine $(\mathrm{CMH})$ as previously described (30). Segments were then frozen in liquid nitrogen, and ESR measurement of superoxide was performed using an EMX ESR spectrometer (Bruker, Karlruhe, Germany) with the following ESR settings: field sweep, $80 \mathrm{G}$; microwave frequency, 9.39 $\mathrm{GHz}$; microwave power, $2 \mathrm{~mW}$; modulation amplitude, $5 \mathrm{G}$; conversion time, $327.68 \mathrm{~ms}$; time constant, $5,242.88 \mathrm{~ms} ; 512$ points resolution; and receiver gain, $1 \times 10^{4}(30)$. The formation of $\mathrm{CM}$-nitroxide was normalized to the dry weight of the lung tissue.

\section{Statistical Analysis}

In all experiments data were analyzed with Student's $t$ test to determine the significance of treatment effects between two groups or with ANOVA when comparing more than two groups. The level of statistical significance was taken as $P<0.05$.

\section{RESULTS}

\section{Characterization of the $\mathrm{CIH}$ Profile}

To induce intermittent hypoxemia in the mouse, we modified a standard, Plexiglas, shoebox box-type cage with a customized lid designed to cycle the $\mathrm{O}_{2}$ tension in the chamber between 21 and $10 \%$. To confirm the reliable achievement of these target oxygen tensions within the animal chamber, an oxygen sensor was placed in the chamber, and the timing of the opening and closing of the door permitting communication with the $\mathrm{N}_{2}$ reservoir and room air was adjusted to accomplish reductions in $\mathrm{O}_{2}$ tension from $21 \%$ to $10 \%$ followed by a return to $21 \%$ oxygen every 90 seconds (data not shown). This profile was designed to mimic alterations in arterial oxygen saturation that might be observed in patients with severe OSA. These alterations in oxygen tension in the animal chamber caused cyclic reductions in oxyhemoglobin saturation in mice lightly anesthetized with isoflurane from $92 \% \pm 0.88$ to $75 \% \pm 0.16$ from four animals as determined with pulse oximetry. A representative tracing from a single mouse is depicted in Figure 1. During exposure to $\mathrm{CIH}$, animals demonstrated obvious tachypnea, but displayed no abnormal behaviors and gained weight over the course of the 8 -week study comparable with that gained by controls (Control $=$ $28.6 \pm 0.6$ versus $\mathrm{CIH}=28.7 \pm 0.4 \mathrm{~g}, n=20$ ).

\section{CIH-Induced Pulmonary Hypertension}

To assess the impact of this $\mathrm{CIH}$ exposure regimen on pulmonary hemodynamics, direct measurements of right ventricular systolic pressure (RVSP) were obtained. As illustrated in Figure 2A, compared with control, exposure to $\mathrm{CIH}$ caused modest but significant increases in RVSP (Control $=26.3 \pm 0.4$ versus $\mathrm{CIH}=$ $32.4 \pm 1.3 \mathrm{~mm} \mathrm{Hg}$ ). To determine the impact of alterations in pulmonary pressures on cardiac mass, RV and LV plus $S$ weights were measured. Compared with Control conditions, $\mathrm{CIH}$ for 8 weeks increased the weight of the free wall of the right ventricle in $\mathrm{C} 57 \mathrm{Bl} / 6$ mice, indicating right ventricular hypertrophy, but had no effect on the weight of the left ventricle plus septum (data not shown). The presence of right ventricular hypertrophy in C57Bl/6 mice was further supported by calculations of the ratio of $\mathrm{RV}: \mathrm{LV}+\mathrm{S}$ weight (Figure $2 \mathrm{~B}$ ). To examine the temporal onset of right ventricular hypertrophy in this model, echocardiography was performed before and at 2-week intervals during $\mathrm{CIH}$ exposure. These results demonstrated that there were no significant differences between $\mathrm{CIH}$ and Control mice with respect to

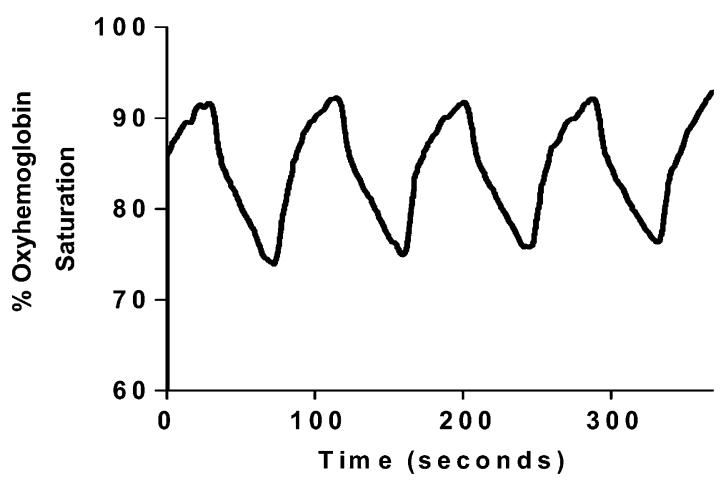

Figure 1. Chronic intermittent hypoxia $(\mathrm{ClH})$-induced alterations in hemoglobin oxygen saturation. A representative tracing of fluctuations in percent oxyhemoglobin saturation $(92 \% \pm 0.88$ to $75 \% \pm 0.16, n=$ 4) detected with pulse oximetry from a single mouse during $\mathrm{CIH}$ exposure is presented. 

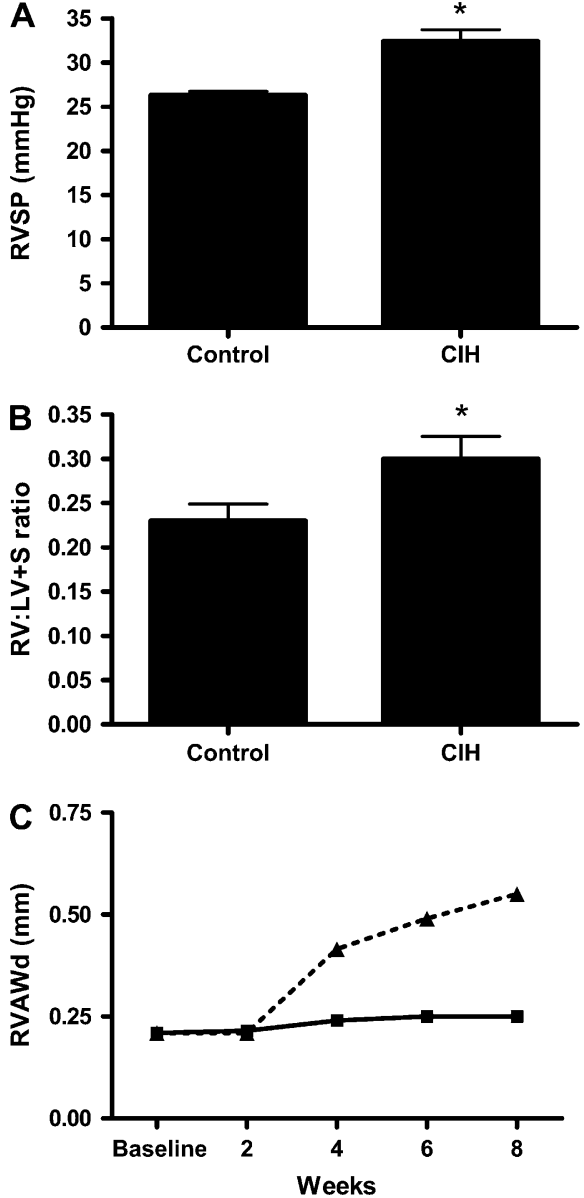

Figure 2. Exposure to $\mathrm{CIH}$ caused pulmonary hypertension. $\mathrm{C} 57 \mathrm{BI} / 6$ mice were exposed to Control (C) or $\mathrm{ClH}$ conditions for 8 weeks. $(A)$ Before mice were killed, a pressure transducing catheter was inserted into the right jugular vein of selected Control and $\mathrm{ClH}$ mice and advanced into the right ventricle, where measurements of systolic pressures were obtained. Data are expressed as mean right ventricular systolic pressure (RVSP) \pm SEM from three to four animals. ${ }^{*} P<0.05$ versus control. $(B)$ Hearts were dissected and weighed at the time of killing. Each bar represents the mean \pm SEM right ventricle: left ventricle + septum (RV:LV+S) weight ratio from 5 to 10 animals. ${ }^{*} P<0.05$ versus Control. (C) Echocardiography was used to assess right ventricle anterior wall thickness before $\mathrm{CIH}$ exposure (baseline) and at 2-week intervals for 8 weeks during exposure to Control (squares, solid line) or $\mathrm{CIH}$ (triangles, dotted line) conditions. Each point represents the mean right ventricular anterior wall thickness from two animals.

LV chamber dimensions, LV posterior wall thickness, LV wall motion, and LV fractional shortening (data not shown). In contrast, the RV anterior wall thickness increased in the CIH group as compared with the Control group (Figure 2C). Serial echocardiograms revealed that RV hypertrophy developed between the second and fourth week of CIH exposure (Figure 2C). The $\mathrm{RV}$ systolic function was grossly normal based on wall motion analysis during two-dimensional imaging, and no significant valvular regurgitation was detected at any time point in Control or $\mathrm{CIH}$ mice (data not shown).

\section{CIH-Induced Pulmonary Vascular Remodeling}

To examine the impact of $\mathrm{CIH}$ on the distribution of muscular layers in small pulmonary vessels, the number of $\alpha-$ SMA-positive
A

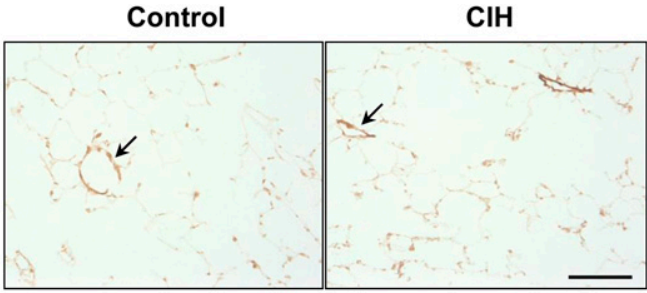

B

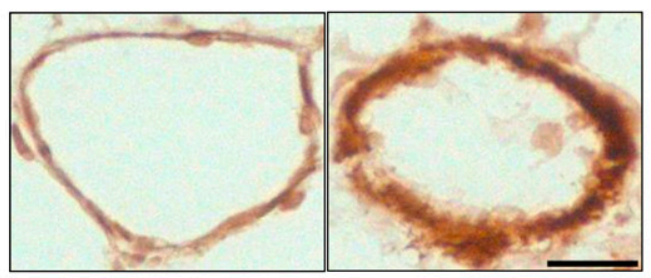

C
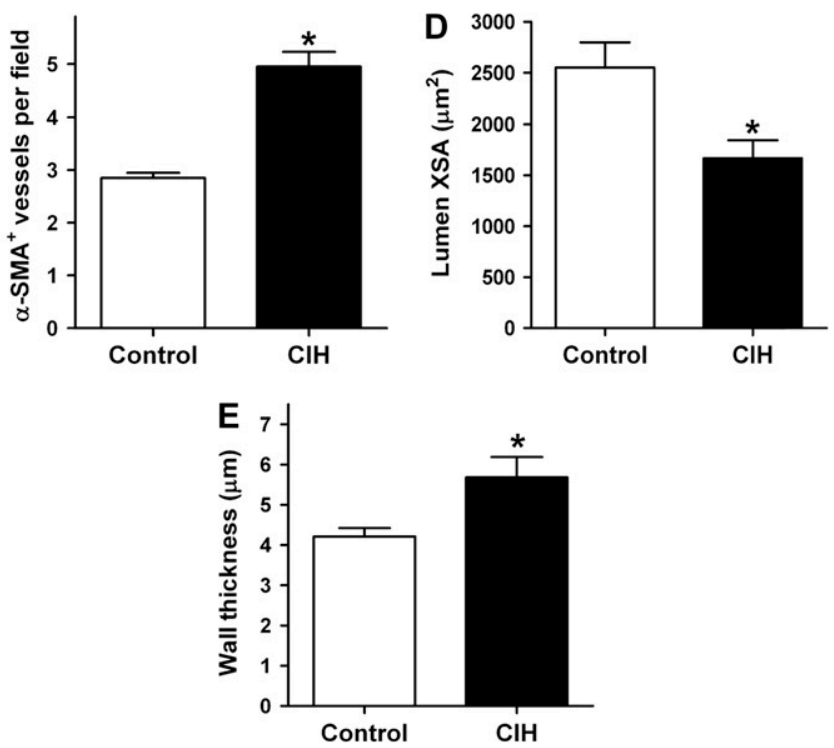

Figure 3. Exposure to $\mathrm{CIH}$ caused pulmonary vascular remodeling. $(A)$ Representative immunostaining of lungs from mice exposed to Control (left) or $\mathrm{ClH}$ (right) conditions. Sections from $\mathrm{ClH}$ mice showed increased $\alpha$-SMA-positive blood vessels. Dark brown staining indicated by the arrowheads are $\alpha$-SMA-positive blood vessels. Bar $=100 \mu \mathrm{m}$. (B) Higher magnification of representative $\alpha$-SMA-stained blood vessels from Control (left) and $\mathrm{CIH}$ (right) lungs showing increased wall thickness and decreased lumenal area due to $\mathrm{CIH}$. Bar $=20 \mu \mathrm{m}$. (C) Quantification of the number of $\alpha$-SMA-positive acinar blood vessels from Control and $\mathrm{CIH}$ groups. Each bar represents the mean \pm SEM number of $\alpha$-SMA-positive blood vessels counted at $\times 10$ magnification in two to four sections from each of five animals per group. (D) Lumenal cross-sectional area (XSA) of $\alpha$-SMA-positive acinar blood vessels from Control and $\mathrm{CIH}$ groups. Each bar represents the mean \pm SEM lumen cross-sectional area (XSA) of $\alpha$ SMA-positive acinar blood vessels in $\mu \mathrm{m}^{2}$. (E) Wall thickness of acinar blood vessels from Control and $\mathrm{ClH}$ groups. Each bar represents the mean \pm SEM wall thickness of $\alpha$-SMA-positive acinar blood vessels in $\mu \mathrm{m} .{ }^{*} p<0.05 ; n=5$ each group.

vessels was counted per microscopic field. Compared with Controls, $\mathrm{CIH}$ increased the number of $\alpha-$ SMA-positive profiles observed per microscopic field (Figures 3A and 3C). Morphometric analyses of vascular profiles $(<100 \mu \mathrm{m}$ lumenal diameter $)$ from $\alpha-$ SMA-stained lung sections demonstrated that $\mathrm{CIH}$ reduced the lumenal cross-sectional area of $\alpha$-SMA-positive vessels (Figure 3D), and increased the degree of vessel muscularization measured as the thickness of the vascular wall (Figures $3 \mathrm{~B}$ and $3 \mathrm{E}$ ). 

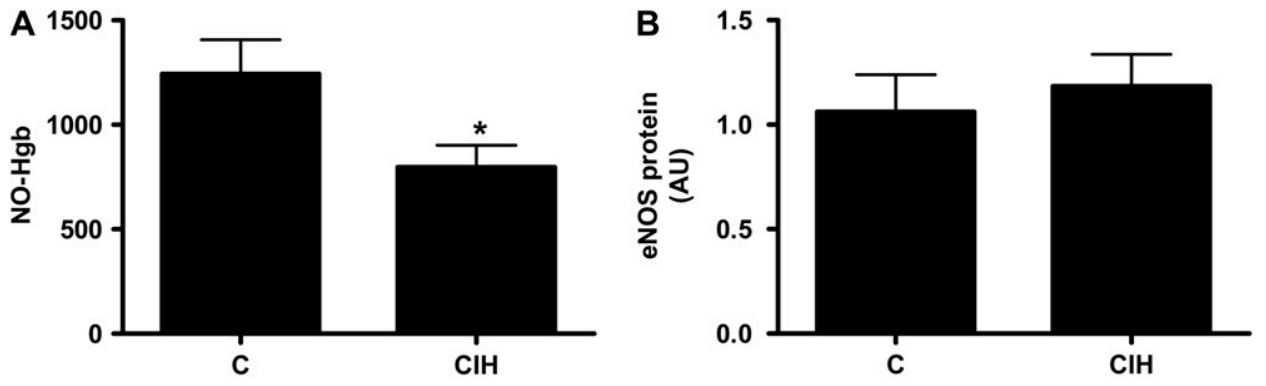

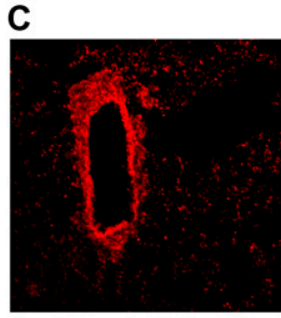

$\mathrm{C}$

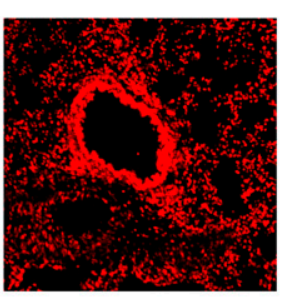

$\mathrm{ClH}$

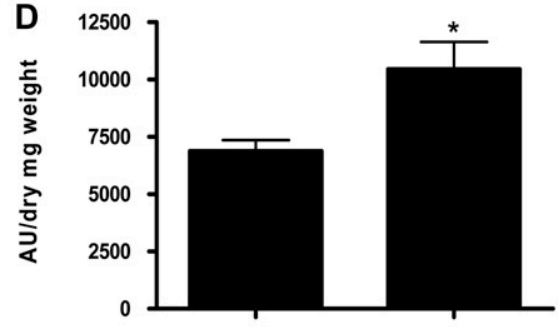

C

$\mathrm{CIH}$
Figure 4. $\mathrm{CIH}$ decreases nitrosyl-hemoglobin formation without altering eNOS expression and increases superoxide production in lung tissue. Mice were exposed to Control or $\mathrm{ClH}$ conditions for 8 weeks. $(A)$ Red blood cells were isolated from a left ventricular puncture and subjected to ESR analysis of NO-Hgb. Each bar represents the mean NO-Hgb level \pm SEM in arbitrary units from 9 to 10 animals. ${ }^{*} P<0.05$ versus Control. (B) Whole lung homogenates were collected and analyzed for eNOS protein expression using Western blotting. Each bar represents the mean eNOS protein expression normalized to actin \pm SEM in arbitrary densitometric units from five to six animals. (C) Lungs were perfused blood free, perfused with $\mathrm{OCT}$, and frozen at $-80^{\circ} \mathrm{C}$ on the day of killing. Thirtyfive-micrometer-thick sections were then prepared and stained with dihydroethidium $(10 \mu \mathrm{M})$. Sections were examined with a fluorescence microscope. Representative images are shown from Control and CIH lungs $(n=5)$. Magnification: $\times 40$. (D) Fresh lung tissue was incubated for 60 minutes in $\mathrm{CMH}$ at $37^{\circ} \mathrm{C}$, then subjected to ESR analysis of superoxide production. Each bar represents the mean formation of 3-methoxycarbonyl-proxyl (CM)-nitroxide normalized to the dry weight of lung tissue \pm SEM from five to six animals in each group. ${ }^{*} P<0.05$ versus control.

\section{CIH Decreased Vascular NO Bioavailability and Increased Superoxide Production in Lung Tissue}

$\mathrm{NO}-\mathrm{Hgb}$ is a global indicator of vascular NO production and has been employed as an index of bioavailable NO (28). The hemoglobin of red blood cells acts as a spin trap by binding to $\mathrm{NO}$ and forming a complex that is detectable using ESR spectroscopy (28). As illustrated in Figure 4A, compared with Control animals, CIH exposure decreased NO-Hgb levels nearly $50 \%$. However, reduction in bioavailable NO was not explained by reductions in lung eNOS protein expression (Figure 4B). The reduction in bioavailable $\mathrm{NO}$ in $\mathrm{CIH}$ mice was associated with increased $\mathrm{O}_{2}^{-\cdot}$ production in the lung. In frozen tissue sections treated with DHE, the intensity of fluorescence is proportional to the amount of $\mathrm{O}_{2}^{-}$produced (31). The representative images shown in Figure 4C illustrate increased intensity of red fluorescence in lungs from $\mathrm{CIH}-$ exposed animals providing qualitative evidence of increased superoxide production. Furthermore, as illustrated in Figure 4D, CIH exposure increased intracellular superoxide production by nearly $25 \%$ compared with control animals as determined by ESR spectroscopy.

\section{CIH Increased Nox4 and p22phox in Lung Tissue}

The NADPH oxidase complex is an important source of superoxide production in lung tissue (29). Exposure to chronic continuous hypoxia specifically increased expression of the Nox4 NADPH oxidase subunit in lung tissue (23). Animals exposed to $\mathrm{CIH}$ for 8 weeks demonstrated increases of both Nox 4 and $\mathrm{p} 22$ phox protein expression in lung tissue (Figures 5A and 5C). Moreover, these increased protein levels were associated with corresponding increases in Nox4 and p22phox mRNA (Figures 5B and 5D). Analysis of additional NADPH oxidase subunits including
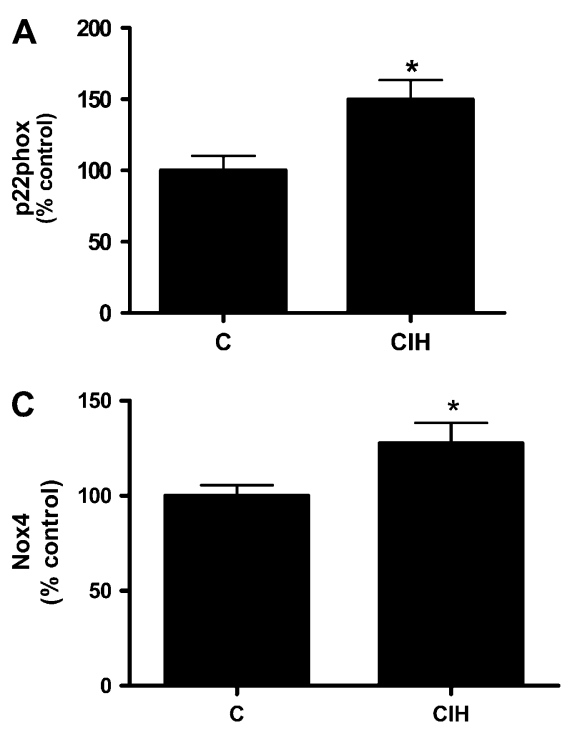
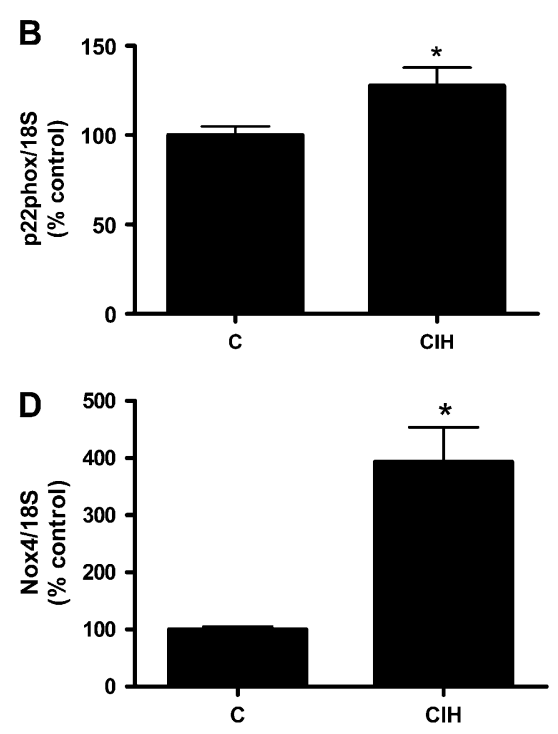

Figure 5. $\mathrm{CIH}$ increased NADPH oxidase subunits, Nox4 and p22phox, levels in lung tissue. After 8 weeks of Control and $\mathrm{ClH}$ exposure, mice were killed, and lung tissue was homogenized. (A) Homogenized lung tissue was immunoprecipitated with antibodies directed against the p22phox subunit of the NADPH oxidase complex. Each bar represents the mean densitometric intensity expressed as percent control \pm SEM from nine animals in each group. ${ }^{*} P<0.05$ versus control. $(B)$ Quantification of p22phox and 18S RNA was performed using CDNA amplification and real-time PCR. Each bar represents the mean copy number per $10^{9}$ copies $18 \mathrm{~S}$, expressed as percent control \pm SEM from eight to nine animals in each group. ${ }^{*} P<$ 0.05 versus Control. (C) Homogenized lung tissue was analyzed for the Nox4 subunit of NADPH oxidase complex. Each bar represents the mean densitometric intensity expressed as percent control \pm SEM from five to six animals in each group. ${ }^{*} P<0.05$ versus wild-type control. (D) RNA was extracted from lung tissue, and real-time PCR performed for Nox4 and 18S. Each bar represents the mean relative expression of Nox4/18S, expressed as percent control \pm SEM from five animals in each group. ${ }^{*} P<0.05$ versus Control. 
p47phox, p67phox, and gp91phox revealed no CIH-induced alterations in levels of these subunits (data not shown), suggesting that the effect of $\mathrm{CIH}$ is specific for Nox4 and p22phox.

\section{CIH Increased PDGFR $\beta$ and Akt Activity}

Because PDGF has been implicated in the pathogenesis of pulmonary hypertension (32-34), we examined the effect of $\mathrm{CIH}$ exposure on PDGFR $\beta$ expression and activity. Exposure to $\mathrm{CIH}$ for 8 weeks significantly increased PDGFR $\beta$ phosphorylation, and these $\mathrm{CIH}$-induced alterations were associated with increased activation and phosphorylation of the downstream effector kinase, Akt (Figures 6A and 6B).

\section{Attenuation of $\mathrm{CIH}$-Induced Pulmonary Hypertension and Associated Signaling Alterations in gp91phox Knockout Mice}

To further characterize the role of NADPH oxidase in CIHinduced pulmonary hypertension, mice lacking the gp91phox NADPH oxidase subunit were exposed to Control or $\mathrm{CIH}$ conditions. As illustrated in Figure 7A, in contrast to the findings in Figure 2B in wild-type mice, gp91phox knockout mice did not develop right ventricular hypertrophy in response to $\mathrm{CIH}$. Furthermore, in contrast to $\mathrm{CIH}$-induced increases in $\mathrm{RV}$ wall thickness in wild-type mice (Figure 2C), there was no increase in RV anterior wall thickness as determined by echocardiography in the mice lacking the gp91phox NADPH oxidase subunit exposed to $\mathrm{CIH}$ (Figure 7B). Similarly, CIHinduced increases in Nox4 protein levels, and increases in PDGFR $\beta$ and Akt activity were completely attenuated in gp91phox knockout mice (Figures 7C-7E). Finally, indices of $\mathrm{CIH}$-induced pulmonary vascular remodeling observed in wildtype animals (Figures 3C-3E) were not observed in gp91phox knockout mice (Figure 7F).

\section{DISCUSSION}

Intermittent hypoxia, hypercapnia, upper airway obstruction, arousals, and alterations in sympathetic tone are all features of OSA-related respiratory events that could affect vascular function. In an attempt to better understand mechanisms of vascular dysfunction related to OSA-associated derangements, the current study employed a mouse model of CIH. To our knowledge, only two previous reports have examined pulmonary vascular effects of $\mathrm{CIH}$ in the mouse $(17,18)$, and comparable to the current results, each study reported the development of pulmonary hypertension. A more recent study examined left ventricular cardiovascular remodeling and systemic vascular effects of $\mathrm{CIH}$ in the mouse (35), but did not extend the study to evaluate the development of pulmonary hypertension. The current study extends these previous reports by demonstrating that $\mathrm{CIH}$-induced alterations in the production of reactive oxygen and nitrogen species play an important role in the pathogenesis of $\mathrm{CIH}$-induced pulmonary hypertension in these models.

Our CIH regimen produced significant pulmonary hypertension evidenced by increased RVSP and right ventricular hypertrophy assessed by cardiac chamber weight analysis and echocardiography. CIH-induced pulmonary hypertension was associated with increased muscularization and remodeling of small pulmonary vessels, consistent with previous reports that $\mathrm{CIH}$ in the mouse leads to pulmonary hypertension. Fagan reported that treating $\mathrm{C} 57 \mathrm{Bl} / 6$ mice with 2-minute cycles of $10 \%$ alternating with $21 \%$ oxygen for 8 hours per day for 4 weeks increased RV systolic pressure, RV mass, and neomuscularization of distal pulmonary vessels, but had no effect on LV mass, although direct measurements of systemic blood pressure were not performed (17). Campen and coworkers (18)
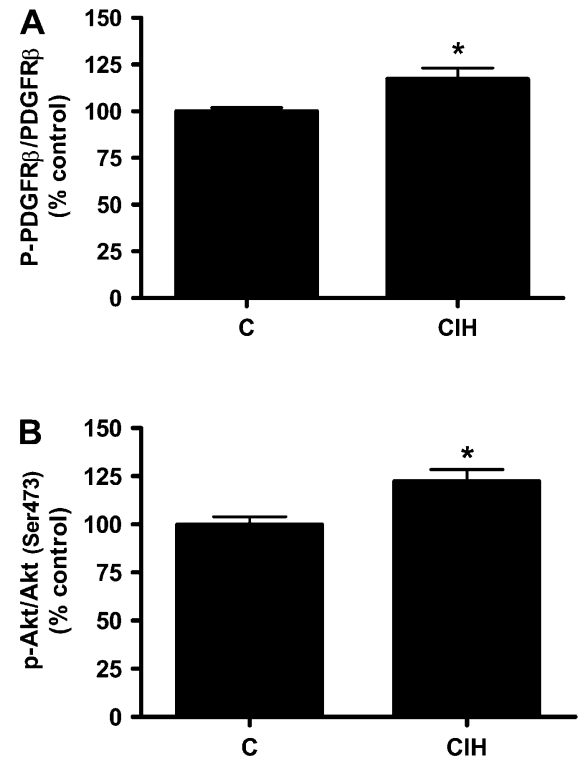

Figure 6. $\mathrm{CIH}$ increased PDGFR $\beta$ and Akt activity. After 8 weeks of Control and $\mathrm{CIH}$ exposure, mice were killed, and lung tissue was homogenized. (A) Phosphorylated PDGFR $\beta$ (P-PDGFR $\beta$ ) was measured in lung homogenates by Western blotting and calculated relative to PDGFR $\beta$ in each sample. Each bar represents the mean P-PDGFR $\beta$ relative to PDGFR $\beta \pm$ SEM as percent control from five to six animals in each group. ${ }^{*} P<0.05$ versus control. (B) Phosphorylated Akt protein levels for serine 473 were measured in lung homogenates by Western blotting and calculated relative to Akt in each sample. Each bar represents mean p-Akt levels relative to Akt \pm SEM as \% Control from five to six animals. ${ }^{*} P<0.05$ versus control.

reported that $\mathrm{C} 57 \mathrm{Bl} / 6$ mice exposed to a 5-week regimen of 60 -second cycles of $21 \%$ alternating with $5 \%$ oxygen for 12 hours per day did develop significant increases in both systemic and pulmonary vascular pressures but more prominent right than left ventricular hypertrophy. These findings emphasize that additional studies will be required to determine which aspects of $\mathrm{CIH}$ (duration, frequency, or severity) best predict vascular dysfunction in the systemic or pulmonary vascular beds.

Our study extends previous reports to examine potential mechanisms by which $\mathrm{CIH}$ produces pulmonary hypertension in the mouse. $\mathrm{CIH}$ caused significant reductions in bioavailable NO that could not be ascribed to $\mathrm{CIH}$-induced reductions in the expression of endothelial nitric oxide synthase, the predominant source of intravascular NO production. $\mathrm{CIH}$ also increased superoxide production in lung parenchyma in our model (Figures 4C and 4D). Because superoxide reacts with NO to form the potent oxidant, peroxynitrite (25), thereby reducing bioavailable NO, we postulate that enhanced superoxide generation in response to $\mathrm{CIH}$ constitutes an important mechanism of vascular dysfunction. The NADPH oxidase complex is an important source of superoxide generation, and mounting evidence suggests that chronic hypoxia-induced activation of NADPH oxidase plays an important role in pulmonary hypertension in the mouse (20). For example, C57Bl/6 mice exposed to chronic continuous hypoxia $(10 \%$ oxygen) for 3 weeks demonstrated increased superoxide generation in pulmonary arteries and increased right ventricular pressure and pulmonary arterial medial wall thickness (22). These chronic hypoxiainduced derangements were completely attenuated in similarly treated gp91phox knockout mice. A more recent report has clarified that chronic continuous hypoxia specifically increased expression of the Nox4 NADPH oxidase subunit (23). Similar 

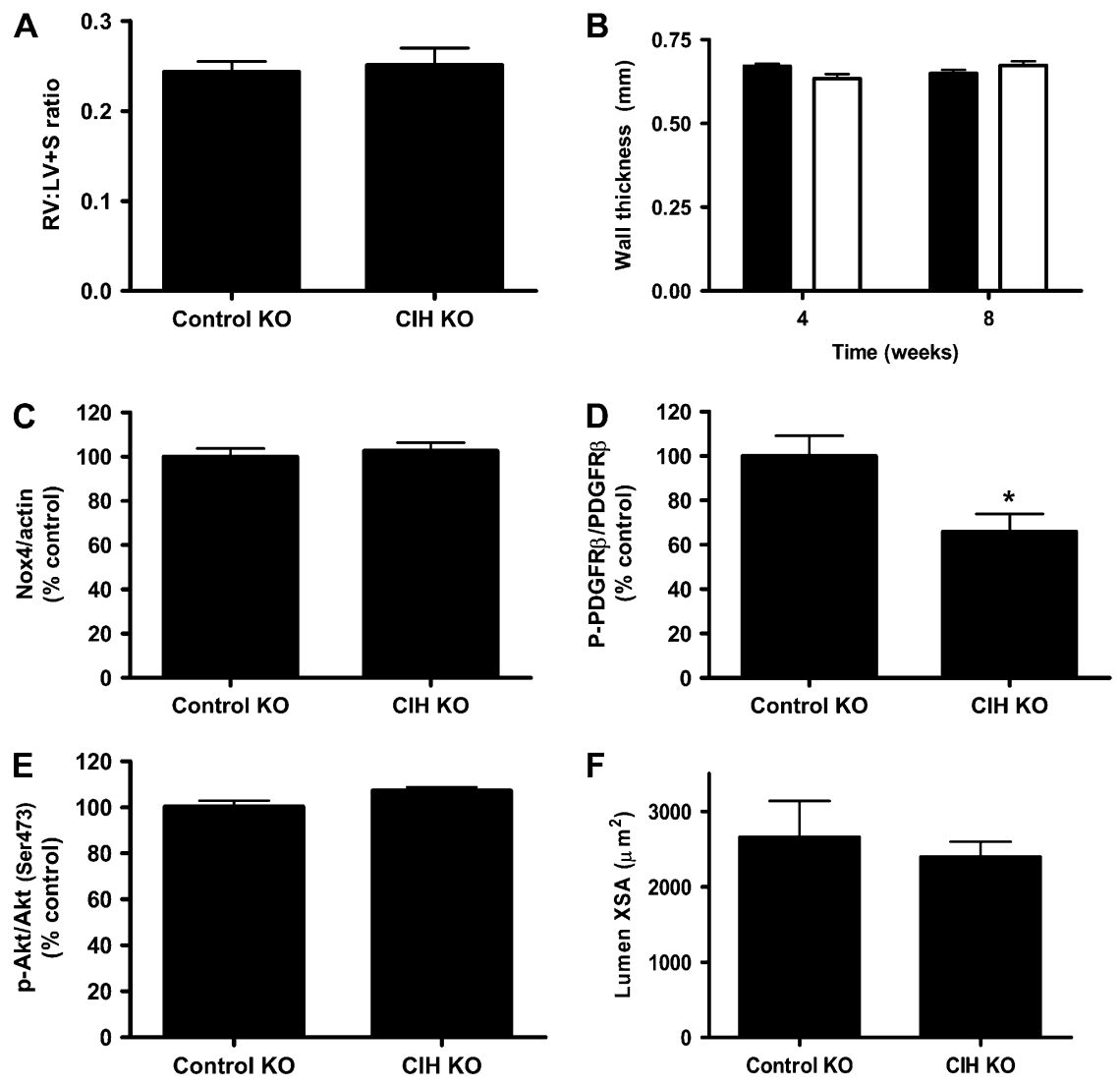

Figure 7. Attenuation of $\mathrm{ClH}$-induced pulmonary hypertension and modulation of associated alterations in gp91phox knockout mice. Mice lacking the gp91phox NADPH oxidase subunit (KO) were exposed to Control or $\mathrm{CIH}$ conditions for 8 weeks as previously described. $(A)$ Ratios of the weight of the right ventricle (13) to the weight of the left ventricle (26) plus septum (S) were calculated and are presented as mean \pm SEM from five animals. (B) Echocardiograms were acquired as previously described at baseline and at 2-week intervals for 8 weeks. Each bar represents the mean \pm SEM of right ventricle anterior wall thickness from four animals per group at 4-week and 8-week time intervals. Solid bars, control KO; open bars, $\mathrm{CIH}$ KO. (C) Homogenized lung tissue was analyzed for the Nox4 subunit of NADPH oxidase complex. Each bar represents the mean densitometric intensityrelative to actin in the same sample \pm SEM expressed as percent control from six to eight animals in each group. (D) Phosphorylated PDGFR $\beta$ was measured in lung homogenates by Western blotting and calculated relative to PDGFR $\beta$ in each sample. Each bar represents the mean P-PDGFR $\beta$ per PDGFR $\beta \pm$ SEM as percent control from six to seven animals in each group. ${ }^{\star} P<0.05$ versus control KO. (E) Phosphorylated Akt protein levels for serine 473 were measured in lung homogenates by Western blotting and calculated relative to Akt in each sample. Each bar represents mean p-Akt levels \pm SEM as \% control from seven to eight animals. ( $F$ ) Lumenal cross-sectional area (XSA) of

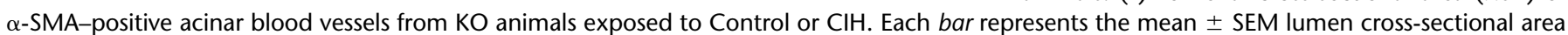
(XSA) of $\alpha$-SMA-positive acinar blood vessels in $\mu \mathrm{m}^{2}(n=4)$.

to prior reports, we have demonstrated that $\mathrm{C} 57 \mathrm{Bl} / 6$ mice exposed to chronic continuous hypoxia (10\% oxygen) for 3 weeks developed pulmonary hypertension as evidenced by increased right ventricular pressure, right ventricular hypertrophy, and increased pulmonary arterial wall thickness. These chronic hypoxia-induced derangements were associated with increased superoxide generation in lung parenchyma and expression of the Nox4 NADPH oxidase subunit (unpublished data). We therefore examined the impact of $\mathrm{CIH}$ on the expression and activity of NADPH oxidase in the lung. Our results (Figure 5) demonstrate that $\mathrm{CIH}$ increased the expression of the NADPH oxidase subunits, Nox4 and p22phox, at both the mRNA and protein levels. While the Nox4 mRNA levels increased by approximately $300 \%$, the Nox 4 protein levels only increased by $25 \%$. We speculate that hypoxia may have increased degradation of Nox 4 protein resulting in the observed differences between the magnitude of CIH-induced increases in mRNA and protein levels. Furthermore, increases in pulmonary hypertension and Nox4 protein levels (Figure 7) were completely attenuated in CIH-treated gp91phox knockout mice indicating the importance of a gp91phox-based NADPH oxidase in the up-regulation of other NADPH oxidase subunits. These results provide novel evidence for the importance of NADPH oxidase-derived reactive oxygen species in the pathogenesis of CIH-induced pulmonary hypertension.

Several reports have emphasized the importance of PDGF in pulmonary hypertension (32-34). When activated, PDGF receptors stimulate cell migration, proliferation, and survival through subsequent activation of several downstream signaling pathways, including Src, phosphatidylinositol 3 kinase (PI3K), phospholipase $C \gamma$, and Ras. In patients with pulmonary hypertension, PDGFR $\beta$ expression was increased in the lungs, and the PDGF receptor antagonist, imatinib, reversed monocrotalineinduced pulmonary hypertension in rats and hypoxia-induced pulmonary hypertension in mice by inhibiting PDGFR $\beta$ phosphorylation (34). Our findings demonstrate that exposure to $\mathrm{CIH}$ for 8 weeks increased PDGFR $\beta$ activity as well as the activity of its downstream effector, Akt. We observed similar increases in PDGFR $\beta$ phosphorylation and activity after exposure to chronic continuous hypoxia in mice (unpublished data). Collectively, these $\mathrm{CIH}$-induced signaling events are postulated to contribute to the vascular smooth muscle proliferation and remodeling observed in $\mathrm{CIH}$-exposed mice. Consistent with this postulate, PDGFR $\beta$ and Akt activity were completely attenuated in CIH-treated gp91phox knockout mice implicating $\mathrm{CIH}$ induced, NADPH oxidase-derived reactive oxygen species as a critical event in the activation of PDGFR $\beta$ and downstream proliferative signaling cascades. The inhibition of $\mathrm{CIH}$-induced right ventricular hypertrophy and pulmonary vascular remodeling in gp91phox knockout mice further substantiates the importance of NADPH oxidase-derived reactive oxygen species and related signaling events in the pathogenesis of vascular dysfunction and pulmonary hypertension.

There are several important limitations to the current study that deserve additional consideration. First, obstructive sleep apnea is essentially an intermittent asphyxiation resulting not only in intermittent hypoxia, but also hypercapnia. The contributions of hypercapnia to vascular dysfunction associated with OSA have not been fully elucidated. The animals in our study exhibited hyperventilation in response to the hypoxic periods although we did not evaluate the role of CIH-induced alterations in carbon dioxide tension in the development of pulmonary hypertension in our model. In a rat model of $\mathrm{CIH}$, carbon dioxide levels had little impact on the effect of $\mathrm{CIH}$ on 
systemic vascular function (36). In addition, it is possible that $\mathrm{CIH}$, by altering sleep quality and/or duration, could potentially produce proinflammatory or oxidative stress responses leading to alterations in vascular function.

Because of the small size of the pulmonary vasculature in the mouse and the technical difficulty inherent in isolating sufficient pulmonary vascular tissue for analysis, we analyzed superoxide production and NADPH expression in peripheral lung parenchyma and measured $\mathrm{NO}-\mathrm{Hgb}$ as a proxy for direct measurements of NO production by resistance vessels in the lung. This strategy could lead to over- or underestimation of $\mathrm{CIH}$-induced alterations in gene expression and the production of reactive species in pulmonary resistance vessels. However, the heterogeneity of cells comprising the wall of pulmonary vessels along the longitudinal axis of the pulmonary vascular tree has recently been emphasized and presents considerable challenges for any analysis of $\mathrm{CIH}$-induced alterations in gene expression and vascular function (37). Our tissue sampling strategy also prevents discrimination of the contribution of the systemic and pulmonary vascular beds to the observed reductions in bioavailable NO and precise attribution of those reductions to NADPH oxidase activity. Similarly, the thickness of the frozen lung sections used for DHE fluorescence imaging prevents the ability to differentiate specific cellular compartments and only provides qualitative evidence of increased superoxide production in lung tissue. While ESR provides quantitative evidence of increased superoxide generation in lung tissue, this technique is also unable to distinguish between vascular and nonvascular cell types. However, it should be emphasized that Mittal and colleagues (23) demonstrated that the hypoxia-induced increase in Nox4 protein was localized to pulmonary vessels but was also seen in the bronchial and alveolar structures, consistent with our DHE fluorescence images (Figure 4C). Although the current study provides direct evidence that NADPH oxidase contributes to $\mathrm{CIH}$-induced pulmonary hypertension in the mouse, our data does not exclude the participation of other enzymatic or mitochondrial sources of ROS. These findings support previous studies demonstrating that NADPH oxidase plays an important role in the production of pulmonary hypertension in response to chronic hypoxia (20), and that $\mathrm{CIH}$ has been reported to up-regulate the expression and activity of NADPH oxidase in selected regions of the brain in C57B1/6 mice (38). Coupled with the current findings, these reports indicate that $\mathrm{CIH}$-mediated induction of NADPH oxidase expression and activity plays an important role in altered vascular function, suggesting novel potential targets for intervention in OSA-associated vascular derangements.

To our knowledge, this is the first report to demonstrate involvement of NADPH oxidase in CIH-induced oxidative stress in the lung and to associate these changes with direct measurements of reduced NO bioavailability. Furthermore, our findings demonstrate the importance of NADPH oxidasemediated alterations in PDGFR $\beta$ activation and downstream signaling pathways in the pathogenesis of $\mathrm{CIH}$-induced pulmonary hypertension. This report thereby provides novel evidence for a mechanistic connection between $\mathrm{CIH}$-induced alterations in NADPH oxidase-derived ROS, reduced NO bioavailability, and pulmonary hypertension.

Conflict of Interest Statement: None of the authors has a financial relationship with a commercial entity that has an interest in the subject of this manuscript.

\section{References}

1. Young T, Palta M, Dempsey J, Skatrud J, Weber S, Badr S. The occurrence of sleep-disordered breathing among middle-aged adults. N Engl J Med 1993;328:1230-1235.
2. Quan SF, Gersh BJ. Cardiovascular consequences of sleep-disordered breathing: past, present and future: report of a workshop from the National Center on Sleep Disorders Research and the National Heart, Lung, and Blood Institute. Circulation 2004;109:951-957.

3. Peppard PE, Young T, Palta M, Skatrud J. Prospective study of the association between sleep-disordered breathing and hypertension. $N$ Engl J Med 2000;342:1378-1384.

4. Nieto FJ, Young TB, Lind BK, Shahar E, Samet JM, Redline S, D'Agostino RB, Newman AB, Lebowitz MD, Pickering TG. Association of sleep-disordered breathing, sleep apnea, and hypertension in a large community-based study. Sleep Heart Health Study. JAMA 2000;283:1829-1836.

5. Parish JM, Somers VK. Obstructive sleep apnea and cardiovascular disease. Mayo Clin Proc 2004;79:1036-1046.

6. Grote L, Ploch T, Heitmann J, Knaack L, Penzel T, Peter JH. Sleeprelated breathing disorder is an independent risk factor for systemic hypertension. Am J Respir Crit Care Med 1999;160:1875-1882.

7. Lavie P, Herer P, Hoffstein V. Obstructive sleep apnoea syndrome as a risk factor for hypertension: population study. BMJ 2000;320: 479-482.

8. Sanner BM, Doberauer C, Konermann M, Sturm A, Zidek W. Pulmonary hypertension in patients with obstructive sleep apnea syndrome. Arch Intern Med 1997;157:2483-2487.

9. Arias MA, Garcia-Rio F, Alonso-Fernandez A, Martinez I, Villamor J. Pulmonary hypertension in obstructive sleep apnoea: effects of continuous positive airway pressure: a randomized, controlled crossover study. Eur Heart $J$ 2006;27:1106-1113.

10. Shahar E, Whitney CW, Redline S, Lee ET, Newman AB, Javier Nieto F, O'Connor GT, Boland LL, Schwartz JE, Samet JM. Sleepdisordered breathing and cardiovascular disease: cross-sectional results of the Sleep Heart Health Study. Am J Respir Crit Care Med 2001;163:19-25.

11. Kraiczi H, Caidahl K, Samuelsson A, Peker Y, Hedner J. Impairment of vascular endothelial function and left ventricular filling: association with the severity of apnea-induced hypoxemia during sleep. Chest 2001;119:1085-1091.

12. Fung JW, Li TS, Choy DK, Yip GW, Ko FW, Sanderson JE, Hui DS. Severe obstructive sleep apnea is associated with left ventricular diastolic dysfunction. Chest 2002;121:422-429.

13. Bady E, Achkar A, Pascal S, Orvoen-Frija E, Laaban JP. Pulmonary arterial hypertension in patients with sleep apnoea syndrome. Thorax 2000;55:934-939.

14. Alchanatis M, Tourkohoriti G, Kakouros S, Kosmas E, Podaras S, Jordanoglou JB. Daytime pulmonary hypertension in patients with obstructive sleep apnea: the effect of continuous positive airway pressure on pulmonary hemodynamics. Respiration 2001;68:566-572.

15. Marrone O, Bonsignore MR. Pulmonary haemodynamics in obstructive sleep apnoea. Sleep Med Rev 2002;6:175-193.

16. Sajkov D, Wang T, Saunders NA, Bune AJ, McEvoy RD. Continuous positive airway pressure treatment improves pulmonary hemodynamics in patients with obstructive sleep apnea. Am J Respir Crit Care Med 2002;165:152-158.

17. Fagan KA. Selected Contribution: Pulmonary hypertension in mice following intermittent hypoxia. J Appl Physiol 2001;90:2502-2507.

18. Campen MJ, Shimoda LA, O'Donnell CP. Acute and chronic cardiovascular effects of intermittent hypoxia in C57BL/6J mice. $J$ Appl Physiol 2005;99:2028-2035.

19. Weissmann N, Zeller S, Schafer RU, Turowski C, Ay M, Quanz K, Ghofrani HA, Schermuly RT, Fink L, Seeger W, et al. Impact of mitochondria and NADPH oxidases on acute and sustained hypoxic pulmonary vasoconstriction. Am J Respir Cell Mol Biol 2006;34:505-513.

20. Fresquet F, Pourageaud F, Leblais V, Brandes RP, Savineau JP, Marthan R, Muller B. Role of reactive oxygen species and gp91phox in endothelial dysfunction of pulmonary arteries induced by chronic hypoxia. Br J Pharmacol 2006;148:714-723.

21. Weissmann N, Tadic A, Hanze J, Rose F, Winterhalder S, Nollen M, Schermuly RT, Ghofrani HA, Seeger W, Grimminger F. Hypoxic vasoconstriction in intact lungs: a role for NADPH oxidase-derived H(2)O(2)? Am J Physiol Lung Cell Mol Physiol 2000;279:L683-L690.

22. Liu JQ, Zelko IN, Erbynn EM, Sham JS, Folz RJ. Hypoxic pulmonary hypertension: role of superoxide and NADPH oxidase (gp91phox). Am J Physiol Lung Cell Mol Physiol 2006;290:L2-L10.

23. Mittal M, Roth M, Konig P, Hofmann S, Dony E, Goyal P, Selbitz AC, Schermuly RT, Ghofrani HA, Kwapiszewska G, et al. Hypoxiadependent regulation of nonphagocytic NADPH oxidase subunit NOX4 in the pulmonary vasculature. Circ Res 2007;101:258-267. 
24. Li JM, Shah AM. Endothelial cell superoxide generation: regulation and relevance for cardiovascular pathophysiology. Am J Physiol Regul Integr Comp Physiol 2004;287:R1014-R1030.

25. Beckman JS, Koppenol WH. Nitric oxide, superoxide, and peroxynitrite: the good, the bad, and ugly. Am J Physiol 1996;271:C1424C1437.

26. West J, Fagan K, Steudel W, Fouty B, Lane K, Harral J, Hoedt-Miller M, Tada Y, Ozimek J, Tuder R, et al. Pulmonary hypertension in transgenic mice expressing a dominant-negative BMPRII gene in smooth muscle. Circ Res 2004;94:1109-1114.

27. Thule PM, Campbell AG, Kleinhenz DJ, Olson DE, Boutwell JJ, Sutliff RL, Hart CM. Hepatic insulin gene therapy prevents deterioration of vascular function and improves adipocytokine profile in STZ-diabetic rats. Am J Physiol Endocrinol Metab 2006;290:E114-E122.

28. Dikalov S, Fink B. ESR techniques for the detection of nitric oxide in vivo and in tissues. Methods Enzymol 2005;396:597-610.

29. Polikandriotis JA, Rupnow HL, Elms SC, Clempus RE, Campbell DJ, Sutliff RL, Brown LA, Guidot DM, Hart CM. Chronic ethanol ingestion increases superoxide production and NADPH oxidase expression in the lung. Am J Respir Cell Mol Biol 2006;34:314-319.

30. Dikalov S, Griendling KK, Harrison DG. Measurement of reactive oxygen species in cardiovascular studies. Hypertension 2007;49:717-727.

31. Miller FJ Jr, Gutterman DD, Rios CD, Heistad DD, Davidson BL. Superoxide production in vascular smooth muscle contributes to oxidative stress and impaired relaxation in atherosclerosis. Circ Res 1998;82:1298-1305.

32. Barst RJ. PDGF signaling in pulmonary arterial hypertension. J Clin Invest 2005;115:2691-2694.

33. Ghofrani HA, Seeger W, Grimminger F. Imatinib for the treatment of pulmonary arterial hypertension. N Engl J Med 2005;353:1412-1413.

34. Schermuly RT, Dony E, Ghofrani HA, Pullamsetti S, Savai R, Roth M, Sydykov A, Lai YJ, Weissmann N, Seeger W, et al. Reversal of experimental pulmonary hypertension by PDGF inhibition. $J$ Clin Invest 2005;115:2811-2821.

35. Dematteis M, Julien C, Guillermet C, Sturm N, Lantuejoul S, Mallaret M, Levy P, Gozal E. Intermittent hypoxia induces early functional cardiovascular remodeling in mice. Am J Respir Crit Care Med 2008; 177:227-235.

36. Fletcher EC, Bao G, Miller CC III. Effect of recurrent episodic hypocapnic, eucapnic, and hypercapnic hypoxia on systemic blood pressure. J Appl Physiol 1995;78:1516-1521.

37. Stenmark KR, Fagan KA, Frid MG. Hypoxia-induced pulmonary vascular remodeling: cellular and molecular mechanisms. Circ Res 2006;99:675-691.

38. Zhan G, Serrano F, Fenik P, Hsu R, Kong L, Pratico D, Klann E, Veasey SC. NADPH oxidase mediates hypersomnolence and brain oxidative injury in a murine model of sleep apnea. Am J Respir Crit Care Med 2005;172:921-929. 